\title{
A APNEIA E A HIPOPNEIA OBSTRUTIVA DO SONO (SAHOS) ASSOCIADA AO DESENVOLVIMENTO DA DOENÇA CARDIOVASCULAR, DIABETES E HIPERTENSÃO ARTERIAL
}

\author{
Lucas Sant'Ana Castilho' \\ Beatriz Corrêa de Almeida Ribeiro² \\ Caroline Strobino ${ }^{3}$ \\ Nicole Silingovischi Ferandes Faria ${ }^{4}$ \\ Aline Santos Cordeiro ${ }^{5}$
}

Resumo: A Síndrome da Apnéia e Hipopneia Obstrutiva do Sono (SAHOS) é uma forma de distúrbio respiratórios relacionados com o sono, frequentemente associada com obesidade, hipertensão e doença arterial coronariana. O objetivo desse estudo foi fazer um levantamento bibliográfico em periódicos indexados datados de 2001 a 2016, associando o desenvolvimento de doenças cardiovascular, diabetes e hipertenção arterial com SAHOS. Através deste estudo podemos confirmar que a Síndrome da Apnéia e Hipopnéia Obstrutiva do Sono (SAHOS) está relacionada principalmente com o desenvolvimento de doenças cardiovasculares, podendo também se associar a diabetes e hipertensão arterial. Percebemos a necessidade de estudos para melhorias nos tratamentos existentes, e a conscientização na parte clínica já que a SAHOS tem uma alta taxa de morbidade no país.

Palavras-chave: Apneia; SAHOS; Hipertensão; Colesterol; Diabetes.

\footnotetext{
1 Odontologia/Universidade do Vale do Paraíba, Brasil. E-mail: lucas.sc0605@gmail.com.

2 Odontologia/Universidade do Vale do Paraíba, Brasil. E-mail: beatrizca.ribeiro@gmail.com.

3 Odontologia/Universidade do Vale do Paraíba, Brasil. E-mail: carolinestrobino@icloud.com.

${ }^{4}$ Odontologia/Universidade do Vale do Paraíba, Brasil. E-mail: nicoleS2silingovschi@hotmail.com.

5 Odontologia/Universidade do Vale do Paraíba, Brasil. E-mail: aline.s.t@hotmail.com.
} 